\title{
DESIGN OF COMPRESSION MEMBERS FOR DURABILITY
}

General principles of structural elements design for durability and their application in the case of compression members are presented in this paper. The degradation effects of material deterioration due to corrosion cause the loss of member resistance, but the limit states method does not directly reflects design and verification allowing for failures caused by material deterioration. The time-dependent loss of compression member resistance was computed for specific corrosion models to express the time-dependent approximation function taking into account effects of corrosion degradation. Then, the design member resistance could be obtained using buckling member resistance multiplied by a timedependent function considering corrosion degradation effects within the member lifetime.

Keywords: durability, prediction of member resistance, time-dependent corrosion effects.

\section{Introduction}

Present standard methods of the reliability theory are based on verification of the structural reliability from the viewpoint of limit states caused by the permanent, variable and accidental actions. Structural failures and damages caused by environmental actions in the form of various material degradations due to aggressive environment and its changes within bridge lifetime are taking into account indirectly or neither in member design. Therefore, durability of steel structures especially bridges is ensured by choice of an appropriate material resistant against the aggressive environment, using appropriate and verified structural details ensuring to minimize environment influences and enabling inspections and maintenance, by structural redundancy and by the choice of an appropriate corrosion protection system.

The general concept of the structural member design for durability is given in standard [1], but the form is useless for practical applications. The reason is, on one hand, the shortage of information about transfer mechanism by which the environmental influences are transferred into agents acting on the structure to cause damages like corrosion. On the other hand, the processes have stochastic character depending on many random variable parameters, so that the mathematic interpretation for structural design is very complicated.

Generally, two concepts of structural verification for durability should be used. The first approach is based on the concept of the bridge design structural service life $t_{s}$, where the probability shall be verified according to formulae

$$
P_{f}=P\left(t_{S} \leq t_{D}\right) \leq P_{f d}
$$

where $t_{D}$ is the bridge design lifetime, $P_{f}$ is the probability of failure and $P_{f d}$ is the target probability of failure. The second approach is based on the limit state concept design considering effects of environmental actions in the form

$$
P_{f}(t)=P[R(t)-E(t) \leq 0\} \leq P_{f d},
$$

where $E(t)$ represents the random variable time-dependent action effects and $R(t)$ is the random variable time-dependent structural member resistance. $E(t)$ and $R(t)$ are functions of random variables $X_{i}$, which are also time-dependent functions in relation with the models of material degradations.

Because of random variable character of many parameters entering corrosion process, the probability theory and mathematic statistics should be the most appropriate approaches to describe it. At present, several corrosion models are known, which could be generally used for analysis of the corrosion influence on the structural member reliability. Review of the most frequent corrosion models describing loss of material in time is presented in Table 1.

\section{Time-dependent buckling member resistance}

Taking into account the buckling effects and the effects of variable randomness which are influencing the resistance of compression member, the buckling member resistance can be expressed in the form (3), that is a function of many material and geometric random variables

$$
R_{c}=\chi \cdot f_{y} \cdot A_{n} \cdot \varphi_{A} \cdot \prod_{i=b}^{n} \varphi_{i},
$$

\footnotetext{
* Josef Vican, Marian Sykora

Department of Structures and Bridges, Faculty of Civil Engineering, University of Zilina, Slovakia, E-mail: vican@fstav.uniza.sk
} 


\begin{tabular}{|c|c|c|c|}
\hline Author & Mean & Standard deviation & Distribution \\
\hline Southwell-Melchers [2] & $0.084 \mathrm{t}^{0.823}[\mathrm{~mm}]$ & $0.056 \mathrm{t}^{0.823}[\mathrm{~mm}]$ & Normal \\
\hline Frangopol [3] & $0.03207 \mathrm{t}^{0.5}[\mathrm{~mm}]$ & $0.00289 \mathrm{t}^{0.045}[\mathrm{~mm}]$ & Normal \\
\hline Qin-Cui [4] & $1.67\left[1-\exp (-\mathrm{t} / 9.15)^{1.97}\right][\mathrm{mm}]$ & $0.0674\left[1-\exp (-\mathrm{t} / 0.181)^{0.0294}\right][\mathrm{mm}]$ & Normal \\
\hline Guedes Soares [5] & \multicolumn{2}{|c|}{$\mathrm{d}_{\text {corr }}(\mathrm{t})=1.5\left(1-\mathrm{e}^{(-\mathrm{t} / 10)}\right)$} & where $\mathrm{t}$ is time in year[mm] \\
\hline
\end{tabular}

where $\chi$ is the actual value of reduction factor covering buckling effects,

$f_{y}$ is the actual value of the steel yield strength $f_{y}$,

$A_{n}$ is the nominal value of the member cross-sectional area,

$\varphi_{A}$ is the ratio of actual value to nominal value of a member cross-sectional area,

$\varphi_{i}=F_{i} / F_{n i}$ is ratio of actual value to nominal value of $i-$ partial effect on member resistance or material.

Following the Ayrton - Perry's approach, the buckling resistance of the member with initial local bow having shape of the half-wave sinusoidal imperfection and the amplitude of $e_{0}$ can be given as

$$
R_{c}=\left\{\frac{1}{2}\left[f_{y}+\left(1+e_{0} \frac{A}{W}\right) \cdot \sigma_{c r}\right]-\left\{\frac{1}{4}\left[f_{y}+\left(1+e_{0} \frac{A}{W}\right) \cdot \sigma_{c r}\right]^{2}-f_{y} \cdot \sigma_{c r}\right\}^{0.5}\right\} \cdot A_{n} \cdot \varphi_{A},
$$

where $\sigma_{c r}$ is the Euler's buckling stress $\sigma_{c r}=\pi^{2} E / \lambda^{2}$,

$R_{c}$ is the buckling resistance of a compression member,

$\eta$ is the Perry's factor, defined as $\eta=e_{0} / r$,

$r$ is the core abscissa, $r=W \mid A$,

$\lambda$ is the member slenderness,

$E$ is the Young's modulus of elasticity,

$e_{0}$ is the amplitude of equivalent geometric imperfection (amplitude of the half-wave sinusoidal imperfection of member centre line).

Taking into account the random variables influences on member resistance the equation can be rewritten into following formula according to [8]

$$
R_{c}=\left\{\frac{1}{2} \Psi-\left[\frac{1}{4} \Psi^{2}-\frac{\varphi_{f y} \varphi_{E} \varphi_{l}}{\varphi_{A} \cdot \overline{\lambda_{n}^{2}}}\right]^{0.5}\right\} \cdot \varphi_{A} \cdot A_{n} \cdot f_{y n},
$$

where $\Psi=\varphi_{f y}+\left(1+e_{0 k} \cdot \frac{A n}{W n} \cdot \varphi_{e 0} \cdot \frac{\varphi_{A}}{\varphi_{W}}\right) \frac{\varphi_{E} \cdot \varphi_{1}}{\varphi_{A} \cdot \overline{\lambda_{n}^{2}}}$,

$$
\lambda_{n}=\sqrt{\frac{f_{y n} L_{c r n}^{2} A_{n}}{\pi^{2} E_{n} I_{n}}}
$$

and the basic random variables are for the Ayrton - Perry's approach introduced in the non dimensional forms as a ratio $\varphi_{i}$, when the actual value is divided by nominal value.

$$
\begin{aligned}
& \varphi_{f y}=\frac{f_{y}}{f_{y n}} ; \varphi_{e 0}=\frac{e_{0}}{e_{0 n}} ; \varphi_{E}=\frac{E}{E_{n}} ; \varphi_{A}=\frac{A}{A_{n}} ; \\
& \varphi_{W}=\frac{W}{W_{n}} ; \varphi_{I}=\frac{I}{I_{n}} .
\end{aligned}
$$

In the case of an exact solution, the influence of both geometric and structural imperfections should be taken into account. Because of difficulties to obtain their values (especially structural imperfections), the equivalent geometric imperfection $e_{0}$ allowing for the initial member bow and also the structural imperfections is more often to use. The characteristic value of this imperfection amplitude is expressed by the following equation

$$
e_{0, k}=e_{0, n}=\alpha(\bar{\lambda}-0.2) W_{e l} / A
$$

where $\alpha$ is the imperfection factor for relevant buckling curve, $\bar{\lambda}=\left(f_{y} / \sigma_{c r}\right)^{0.5}$ is the non-dimensional slenderness.

The equation (9) can be rewritten using Ayrton - Perry's formula as follows

$$
\begin{aligned}
& e_{0, k}=\frac{(1-\chi) \cdot\left(1-\bar{\lambda}^{2} \chi\right)}{\chi} \frac{W_{e l}}{A}=(1-\chi) . \\
& \cdot\left(1-\bar{\lambda}^{2} \chi\right) \frac{M_{e l, k}}{\chi \cdot N_{p l, k}}=(1-\chi) \cdot\left(1-\frac{N_{p l, k}}{N_{c r}} \chi\right) \frac{M_{e l, k}}{\chi \cdot N_{p l, k}} .
\end{aligned}
$$

Then, for the design value of the amplitude of the equivalent geometric imperfection, the following equation is valid

$$
e_{0, d}=e_{0, k} \cdot \frac{1-\chi N_{p l, k} /\left(\gamma_{M 1} \cdot N_{c r}\right)}{1-\chi N_{p l, k} / N_{c r}}=e_{0, k} \cdot \frac{1-\bar{\lambda}^{2} \chi / \gamma_{M 1}}{1-\bar{\lambda}^{2} \chi} .
$$

The mean value and the standard deviation of the equivalent imperfection amplitude $e_{0}$ needed for further probabilistic analysis, can be obtained assuming the normal distribution function using solution of equation system

$$
\begin{aligned}
& e_{0, k}=\mu_{e 0}+\beta_{5 \%} \cdot \sigma_{e 0}=\mu_{e 0}+1.645 \cdot \sigma_{e 0} \\
& e_{0, d}=\mu_{e 0}+\beta_{d} \cdot \alpha_{R(E)} \cdot \sigma_{e 0}=\mu_{e 0}+3.8 \cdot 0.8 \cdot \sigma_{e 0}
\end{aligned} .
$$

\section{Parametric study of the time-dependent buckling member resistance considering corrosion degradation}

To determine effects of the corrosion losses on the buckling member resistance, the parametric study of I - cross-sectional member was realized (the results of previous parametric studies for 6 beams realized for bending moment resistance are presented in [9]). All cross-sectional dimensions $b_{f}, t_{f}, h_{w}, t_{w}$ as well as the yield strength $f_{y}$ were considered as random variables according to 
above mentioned studies for cross-section no. 2 which consists of flanges with nominal dimensions $150 \times 16 \mathrm{~mm}$ and web with parameters of $268 \times 6 \mathrm{~mm}$. Standard deviations of the considered cross-sections were determined using the standard for tolerances of web heights and flange widths [6] and according to standard for tolerances of web and flange thicknesses [7] (class A). They were obtained based on the assumption of the normally distributed random variable standard tolerance $a$ and that $95 \%$ of random variable realizations are in interval (so called $2 \sigma$ rule)

$$
\left\langle m_{x}-a ; m_{x}+a\right\rangle .
$$

To determine the buckling resistance of a compression member respecting the time-dependent degradation effects caused by corrosion of its flanges, the above mentioned approach was used. Numerical analysis of the determining time-dependent buckling resistance taking into account the randomness of the cross-sectional dimensions and yield strength was realized using software MATLAB This parametric study was calculated for the non-dimensional slenderness $\lambda_{y}=\lambda_{z}=0.25 ; 0.50 ; 0.75 ; 1.00 ; 1.25$ and 1.50 . The values of random variables were generated by means of Latin Hypercube Sampling (LHS) for 10000 samples considering normal distribution. For all the prediction models of corrosion losses (see Table 1), the time-dependent development of buckling resistance within the design working life of 100 years was computed. The corrosion losses $d_{\text {corr }}$ described by the previously mentioned prob- abilistic models were considered as reduction of flange thickness given by formula $t_{\text {fred }}=t_{f}-d_{\text {corr }}$, where $t_{f}$ is the flange thickness without corrosion effects.

Degradation function $F(t)$ was derived as the ratio of timedependent resistance $R(t)$ of degraded member and resistance $R_{0}$ at the beginning of bridge member exploitation.

$$
F(t)=R(t) / R_{0}
$$

For further general use, the degradation function $F(t)$ has been approximated by means of regression techniques based on the function given by formula (15) using MATLAB software environment.

$$
F(t)=a \cdot e^{-b . t}+c
$$

Mean values of computed buckling resistances taking into account the random variables effects can be seen in Fig. 1. In Fig. 2 , the mean values of compression member buckling resistances in dependence on non-dimensional slenderness $\bar{\lambda}_{y}$ and $\bar{\lambda}_{z}$ for relevant corrosion models are shown.

The strong correlation between fitted function and actual degradation function expressed by means of regression coefficient Rsquare was obtained. From this study follows, that value $\boldsymbol{b}$ in formula
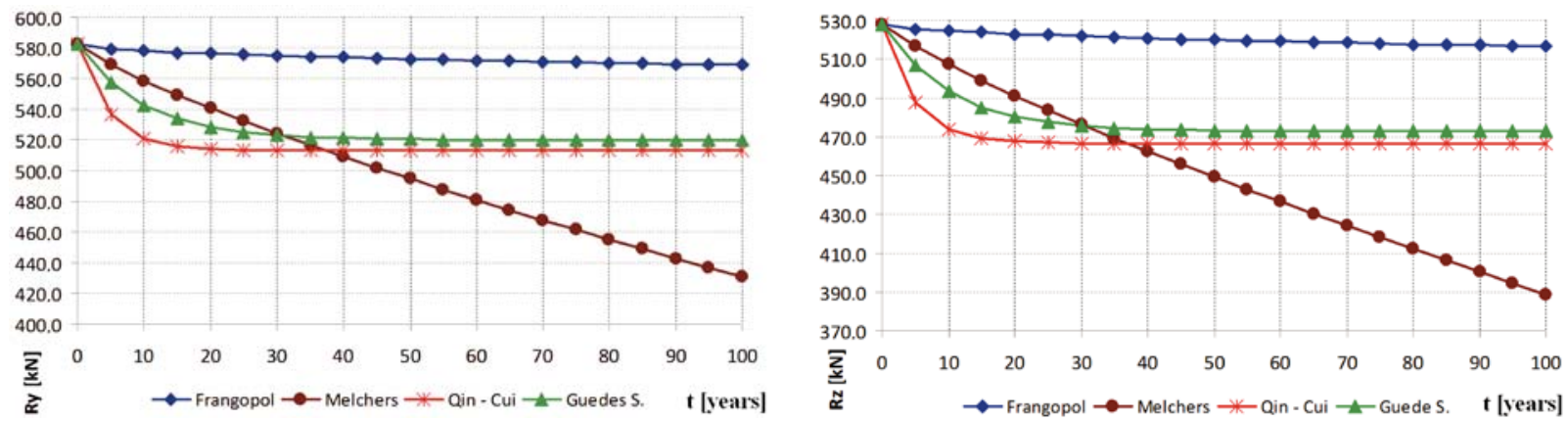

Fig. 1 Time-dependent development of mean values of buckling resistances for non-dimensional slenderness $\lambda=1.50$ ( $R_{y}$ on the left, $R_{z}$ on the right)
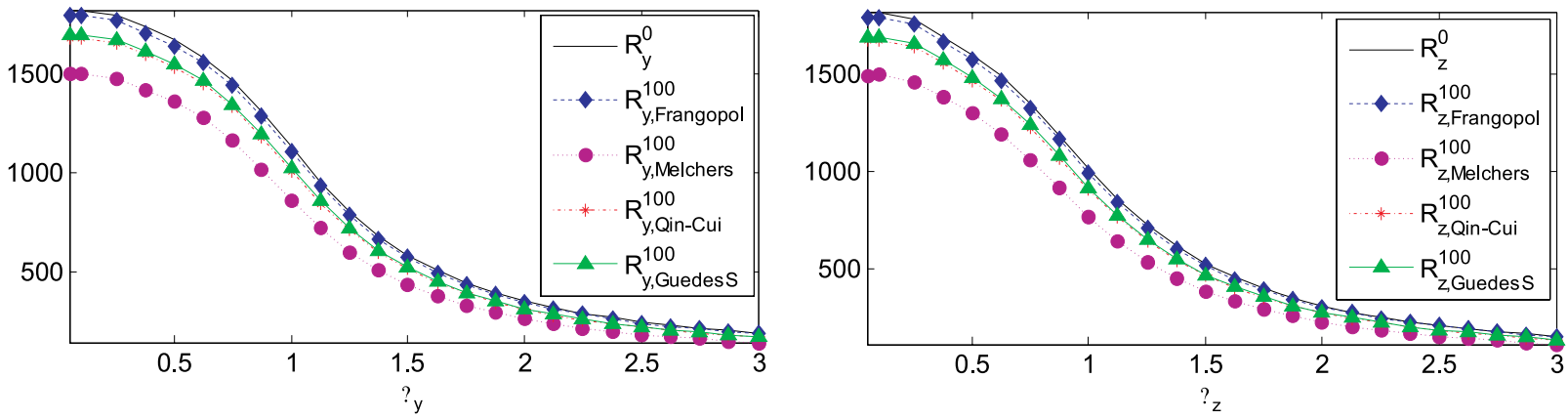

Fig. 2 The buckling resistances $R y$ and $R z$ in dependence on non-dimensional slenderness $\bar{\lambda}$ for cross-section no. 2 (in zero time and after 100 years) 
(15) remains for relevant corrosion model unified and constants $a, c$ are only changing. This fact leads to an idea to apply the relevant corrosion model in dependence on environmental condition using known "degradation constant $\boldsymbol{b}$ " and the "shape constants $\boldsymbol{a}$, $c$ " should be computed in dependence on cross-sectional characteristics. For practical use, the degradation constant $\boldsymbol{b}$ is supposed to represent time-dependent corrosion progress depending on the relevant corrosion prediction model and constants $\boldsymbol{a}, \boldsymbol{c}$ are defining the cross-sectional characteristics.

There was also observed in the study that the values of all approximation constants are identical for both relevant buckling modes $y$ or $z$ and, therefore, the buckling mode about axis $y$ has only been investigated in following studies.

For better understanding of this problem and due to effort to get a general solution, the second parametric study for $2000 \mathrm{I}$ - beams with random cross-sectional characteristics was realized allowing for maximum non-dimensional slenderness $\bar{\lambda} \leq 3.0$. This study brought the knowledge that for compression members the degradation constant $\boldsymbol{b}$ has the same value as was derived for members subjected to bending [9]. The possibility to use the equation for determining the shape constant $\boldsymbol{a}$ in the form of (16) derived for bending members was also proved but with the limited validation for compression members having non-dimensional slenderness $\bar{\lambda} \leq 0.2$.

Sensitivity analysis for 2000 I cross sections, $\lambda=0.00$
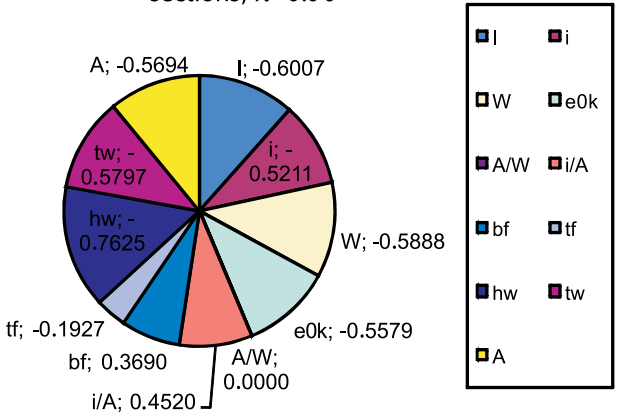

$$
a_{\bar{\lambda} \leq 0.2}=2 b_{f} / A \cdot \alpha_{\text {corr.model }},
$$

where $b_{f}$ is the flange width of I cross-section,

$A$ is the cross-sectional area,

$\alpha_{\text {corr.model }}$ is the corrosion constant for relevant corrosion model given in Table 2 .

For member with non-dimensional slenderness $\bar{\lambda}>0.2$, the determination of shape constant $a$ is more complicated because the value of this constant varies in dependence on the actual nondimensional slenderness. The random variable cross-sectional characteristics and the time are other complicating factors. Therefore, the sensitivity analysis for realized second parametric study of 2000 I- beams was worked up. The results of this analysis are shown in Fig. 3.

Using results of sensitivity analysis, the numerically determined dependence of shape constant $\boldsymbol{a}$ on the non-dimensional slenderness $\bar{\lambda}$ was obtained as a ratio of shape constant $\boldsymbol{a}(\bar{\lambda})$ for arbitrary relative slenderness and value of shape constant $a(\bar{\lambda}=0)$ for compression members with zero non-dimensional slenderness. The final dependence determined for 2000 I- beams and for corrosion model according to Frangopol is shown in Fig. 4.

In the frame of exact solution the function describing the dependence of shape constant $\boldsymbol{a}$ on non-dimensional slenderness $\bar{\lambda}$

\section{Sensitivity analysis for $2000 \mathrm{I}$ cross -} sections, $\lambda=300$

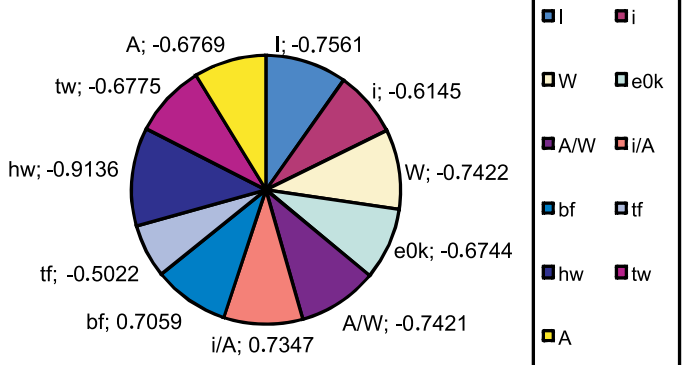

Fig. 3 Sensitivity analysis for 2000 I cross-sections in dependence on non-dimensional slenderness $\bar{\lambda}_{y}$

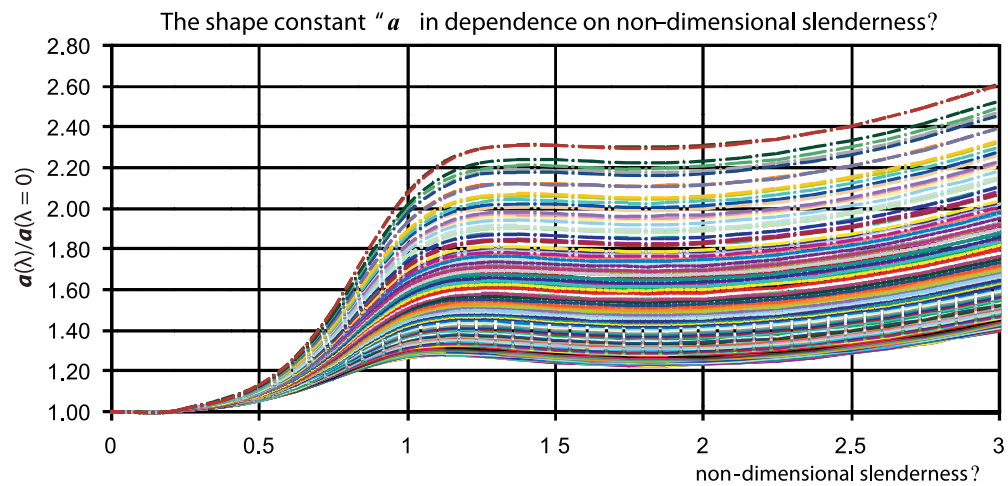

Fig. 4 The shape constant a in dependence on non-dimensional slenderness $\bar{\lambda}$ 
was not found due to very complicated multi-dimensional problem depending on 4 variables requiring to work with large number of data. To simplify the problem, such a case was verified, where the buckling resistance is computed in every year and for all nondimensional slenderness range using the value of constant $\boldsymbol{a}$ valid for the case of pure compression member, i. e. $\bar{\lambda} \leq 0.2$. The results of this simplification were compared to the results obtained by the numerical calculations and the analysis results showed that the progress of ratios of buckling resistance mean values is almost linear in time. Nevertheless, the deviation between exact and simplified solution is increasing in dependence on time. But for common corrosion model, the deviation increase does not exceed the value of $4 \%$. Providing that this deviation increase could be neglected the resistance ratio in time zero can be used, which is identical for all corrosion models, and the equation for a correction factor $\Delta(\lambda)$ would be obtained in the following form

$$
\begin{aligned}
\Delta(\lambda) & =1.023+0.015 \cdot \cos (2.55 \bar{\lambda})- \\
& -0.07 \cdot \sin (2.55 \bar{\lambda}) .
\end{aligned}
$$

Finally, the time-dependent buckling resistance of the deteriorated compression member at the time $t$ could be obtained using the following formulae

$$
\begin{aligned}
& N_{R d}(t)_{\lambda \leq 0.2}=N_{R d, t=0} \cdot F(t)=A \cdot f_{y} \cdot\left[1+a\left(e^{-b t}-1\right)\right], \\
& N_{R d}(t)=N_{R d, t=0} \cdot F(t)=A \cdot \chi \cdot f_{y} / \Delta(\lambda) . \\
& \cdot\left[1+a\left(e^{-b t}-1\right)\right], \\
& a=2 \cdot b_{f} / A \cdot \alpha_{\text {corr.model }},
\end{aligned}
$$

where the value of the constant $\boldsymbol{a}$ is calculated according to (20) while for constants $\boldsymbol{b}, \alpha_{\text {corr }}$. model the values in accordance with table 2 are valid.

Values of corrosion constants.

Table 2

\begin{tabular}{|c|c|c|}
\hline \multirow{2}{*}{ Corrosion model } & \multicolumn{2}{|c|}{ Constant } \\
\cline { 2 - 3 } & $\boldsymbol{b}$ & $\alpha_{\text {corr model }}$ \\
\hline Frangopol & 0.0194 & $3.278 \mathrm{E}-04$ \\
\hline Melchers & 0.0055 & $8.460 \mathrm{E}-03$ \\
\hline Qin - Cui & 0.2158 & $1.655 \mathrm{E}-03$ \\
\hline Guedes Soares & 0.1003 & $1.490 \mathrm{E}-03$ \\
\hline
\end{tabular}

\section{Conclusion}

The member resistance is significantly influenced due to degradation effects in the form of corrosion and therefore the corrosion effects should be taken into account for structural design. This paper presents simplified approach to the determination of the timedependent member resistance. Nevertheless, the above described approach for design of compression members for durability is not worked up for standard using up to now. The more complex stochastic analysis comparing obtained results with experimental ones should be realized.

\section{Acknowledgement}

The paper presents results of the research activities supported by the Slovak Research and Development Agency under the contract No. APVV-20-010005 and by the Slovak Grant Agency, grant No. $1 / 0311 / 09$.

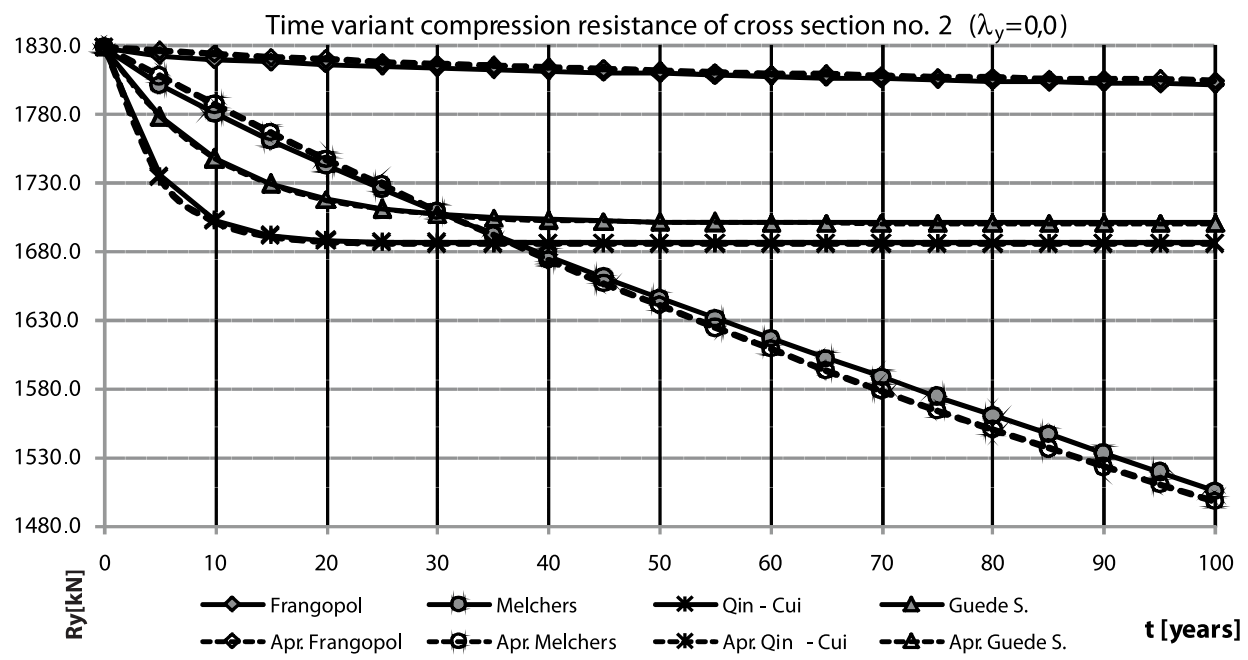

Fig. 5 Comparison of the numerically and according to (18) determined time-dependent member buckling resistance 
Time variant buckling resistance of cross section no. 2 for non

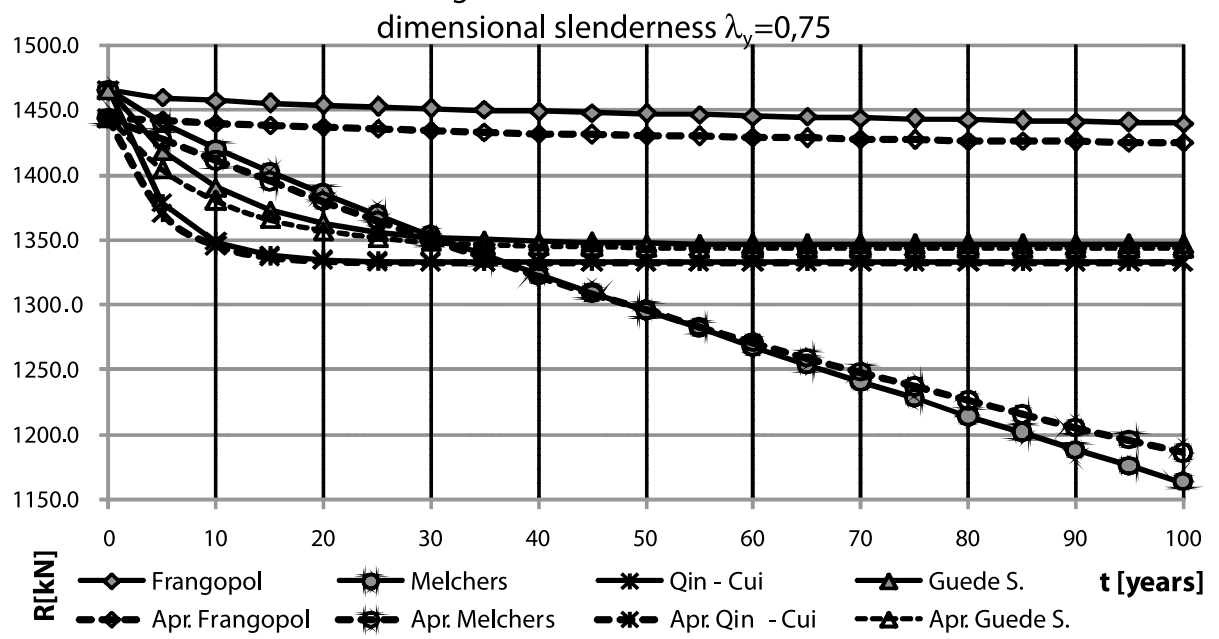

Fig. 6 Comparison of the numerically and according to (19) determined time-dependent member buckling resistance

\section{References}

[1] ISO DIS 13823: General Principles on the Design of structures for Durability. ISO/TC 98/SC 2.

[2] MELCHERS, R. E.: Corrosion Uncertainty Modelling for Steel Structure. Journal of Constructional Steel Research, Elsevier, 1999.

[3] AKGUL, F., FRANGOPOL, D. M.: Lifetime Performance Analysis of Existing Steel Girder Bridge Superstructures. Journal of Structural Engineering v. 130, No. 12, pp. 1875-1888.

[4] QIN, S., CUI, W.: Effect of Corrosion Models on the Time-dependent Reliability of Steel Plated Elements. Marine Structures, Elsevier, 2003

[5] GUEDES-SOARES, C., GARBATOV, Y.: Reliability of Maintained, Corrosion Protected Plates Subjected to Non-linear Corrosion and Compressive Loads. Marine Structures, Elsevier, 1999.

[6] STN P ENV 1090-1: Execution of Steel Structures. Part 1 - General Rules and Rules for Buildings, SUTN, Bratislava, 2001.

[7] STN EN 10029+AC 1998: Tolerances on Dimensions, Shape and Mass for Hot Rolled Steel Plates 3 mm Thick or Above. SUTN, Bratislava, 1998.

[8] PALES, D., SADOVSKY, Z.: On Reliability Verification and Column Buckling. Building Research Journal, vol. 44, 3/1996

[9] VICAN, J., SYKORA, M.: Design of Bridge Structural Members for Durability. In: International Conference VSU' 2008, Sofia 2008, pp. 111-116.

[10] VICAN, J., SYKORA, M.: Design of Compression Members for Durability (in Slovak). In: International Conference 70 Years of FCE STU, Bratislava, 2008. 